Revista de Estudios Histórico-Jurídicos [Sección historia del derecho (público y privado)]

XLI (Valparaíso, Chile, 2019) [pp. 157 - 176]

\title{
La importancia de la Primera Conferencia Regional Americana del Trabajo Realizada En Chile EN 1936
}

[The importance of the First American Regional Conference of Labour held in Chile in 1936]

\author{
Pablo Arellano Ortiz* \\ Organización Internacional del Trabajo, Ginebra, Suiza
}

\begin{abstract}
RESUMEN
El presente trabajo tiene por objeto analizar la Primera Conferencia Regional Americana del Trabajo realizada en Chile en 1936. A nuestro entender dicha reunión marca fuertemente la manera cómo la OIT se relacionará con la región latinoamericana, pero, sobre todo, por la influencia que han tenido, en el desarrollo posterior de la organización, los temas que ahí se trataron. De esta manera en un primer apartado revisaremos algunos antecedentes en relación a la conferencia para luego, en un segundo apartado, referirnos a los temas tratados y su influencia en el desarrollo posterior de la OIT. Finalizaremos el trabajo con algunas reflexiones.
\end{abstract}

\section{Palabras Clave}

Organización Internacional del Trabajo-Chile-derecho internacional público - derecho del trabajo.

\section{AbStRaCt}

The purpose of this work is to analyze the First American Regional Conference on Labor held in Chile in 1936. In our opinion, this meeting strongly marks the way in which the ILO will relate to the Latin American region, but above all because of the influence it has had, in the later development of the organization, the issues that were discussed there. In this way in a first section we will review some background in relation to the conference and then in a second section we will refer to the topics discussed and their influence on the further development of the ILO. We will finish the work with some reflections.

\section{KeYWORDS}

International Labour Organization - Chile - International Public Law Labour Law.

ReCibIDO el 30 de abril de 2019 y aCEPTADO el 28 de junio de 2019.

*Especialista en Derecho del Trabajo, Unidad de Derecho Laboral y Reforma, Departamento de Gobernanza y Tripartismo de la OIT, Ginebra (Suiza); Investigador, Facultad de Derecho, Pontificia Universidad Católica de Valparaíso (Chile). Las opiniones expresadas incumben solamente al autor y no representan necesariamente los puntos de vista de la Organización Internacional del Trabajo (arellano@ilo.org). 


\section{INTRODUCCIÓN}

La Organización Internacional del Trabajo (OIT) se encuentra celebrando este año su primer centenario. Dentro de este periodo la presencia de América Latina, como región, y de Chile, en cuanto a país miembro ha sido muy relevante. En palabras de Humberto Villasmil: "En la ya célebre Parte XIII del Tratado (Artículos 387 a 399) se incluye lo que es hoy, con sus reformas, la Constitución de la OIT de 1919. Lo firmaron 32 Estados, miembros originarios de la Sociedad de las Naciones; de ellos, más de un tercio, once (11) para ser exactos, fueron países latinoamericanos, a la sazón: Bolivia, Brasil, Cuba, Ecuador, Guatemala, Haití, Honduras, Nicaragua, Panamá, Perú y Uruguay. Pero, añadidamente, de los 13 Estados 'invitados a acceder al convenio', seis (6) fueron países Latinoamericanos, a la sazón: Argentina, Chile, Colombia, Paraguay, El Salvador y Venezuela”.

La OIT tiene como principal función el establecer normas internacionales del trabajo: convenios y recomendaciones ${ }^{2}$. Su labor normativa ha sido altamente destacada durante el siglo XX. Así se indicado, por ejemplo, que desde su fundación "ha puesto en marcha unos dispositivos únicos a escala internacional que permiten supervisar el progreso de los Estados Miembros del organismo en la aplicación de las normas internacionales del trabajo, en particular los convenios que han ratificado" . También se ha destacado "el hecho de que la OIT juega un papel importante y activo en la aplicación de los derechos humanos clásicos en el piso de trabajo es algo que generalmente permanece subexpuesto" ${ }^{4}$.

Como señala Yáñez nuestro país no estuvo ajeno a este movimiento social de principios de 1900. De hecho “aprobó una naciente legislación social desde 1906 y un año después creó la Oficina del Trabajo, organismo encargado inicialmente de llevar a cabo la estadística laboral pero que terminó fiscalizando el cumplimiento de esa legislación. En 1919 Chile fue una de las tantas naciones firmantes del protocolo de acuerdo que creaba la OIT, y en 1925, en el contexto de la visita de Albert Thomas, Director de dicho organismo, nuestro país aprobó los decretos que lo ponían a la par con las disposiciones aprobadas en las distintas Conferencias del Trabajo, especialmente la de Washington de 1919"5.

${ }^{1}$ Villasmil Prieto, Humberto, La incidencia de la Organización Internacional del Trabajo en el momento fundacional del derecho del trabajo latinoamericano: unas notas introductorias, en Dialogue. Documento de trabajo, núm.33, Departamento de Relaciones Laborales y de Empleo (Oficina Internacional del Trabajo, Ginebra: OIT, 2011), p. 1.

${ }^{2}$ Véase al respecto, Arellano Ortiz, Pablo, La conformidad de la legislación chilena a las normas internacionales del trabajo de la OIT, en Revista de Derecho de la Universidad Católica de la Santísima Concepción, 24 (junio 2011), pp. 39-60; WalKer Errázuriz, Francisco y Arellano Ortiz, Pablo, Derecho de las relaciones laborales, I: Derecho individual del trabajo (Santiago, Librotecnia, 2016); OIT, Las reglas del juego: Una introducción a la actividad normativa de la Organización Internacional del Trabajo (Ginebra, Oficina Internacional del Trabajo, 2019).

${ }^{3}$ Borvin, Isabelle y ODERo, Alberto, La Comisión de Expertos de la OIT y el progreso de las legislaciones nacionales, en Revista Internacional del Trabajo, 125/3 (2006), p. 233.

${ }^{4}$ Van Der Heijden, Paul, The ILO Stumbling towards Its Centenary Anniversary, en International Organizations Law Review, 15 (2018), p. 204.

${ }^{5}$ Yáñez Andrade, Juan Carlos, Chile y la Organización Internacional del Trabajo (1919- 
A la cabeza de la Oficina del Trabajo creada en Chile en 1920 encontramos a Moisés Poblete, quien fue nombrado por el electo Presidente de la República, Arturo Alessandri, como Director de la Oficina del Trabajo ${ }^{6}$. Quien desde dicha posición juega un rol importante en la relación entre nuestro país y la OIT en sus inicios.

La relación entre la OIT y nuestro país ya ha sido estudiada previamente por diversos autores ${ }^{7}$. En este sentido se ha destacado dos acontecimientos dentro de la historia de la OIT como relevantes en relación con América Latina ${ }^{8}$. El primero de ellos es el paso de David H. Blelloch por Venezuela en 1936, al tratarse de la primera misión enviada por la OIT a un país miembro para prestar asistencia técnica in situ en el marco de la redacción de una legislación del trabajo. El otro evento destacado es la Primera Conferencia Regional Americana de los Estados miembros realizada en 1936 en Santiago de Chile.

Nuestra intención es referirnos a este último evento en particular. Nos gustaría resaltar, bajo una óptica histórico jurídica, la Primera Conferencia Regional Americana del Trabajo de 1936. Ello porque a nuestro entender dicha reunión marca fuertemente la manera cómo la OIT se relacionará con la región latinoamericana, pero sobre todo por la influencia que han tenido, en el desarrollo posterior de la organización, los temas que ahí se trataron. De esta manera en un primer apartado revisaremos algunos antecedentes en relación a la conferencia para luego en un segundo apartado referirnos a los temas tratados y su influencia en el desarrollo posterior de la OIT. Finalizaremos este trabajo con algunas reflexiones.

\section{La Primera Conferencia Regional Americana del Trabajo de 1936: ORÍGENES Y DESARROLLO}

El origen de esta reunión corresponde a la iniciativa del gobierno chileno de proponer una reunión de esta envergadura. Como hemos señalado la relación de Chile con la OIT en sus inicios fue bastante estrecha y ello se quiso profundizar. Así entonces en la Conferencia Internacional del Trabajo del año 1935 el representante gubernamental de Chile, señor García Oldini ${ }^{9}$, realiza formalmente la invitación ${ }^{10}$, la cual fue bien acogida por los otros miembros de la conferencia ${ }^{11}$.

1925). Hacia una legislación social universal, en Revista de Estudios Histórico-Jurídicos, 22 (2000), p. 317.

${ }^{6}$ Como se indica ibíd., p. 324.

${ }^{7}$ Por ejemplo, Yáñez Andrade, Juan Carlos, Chile y la Organización, cit. (n. 5); Arellano Ortiz, Pablo, La conformidad, cit. (n. 2).

${ }^{8}$ Villasmil Prieto, Humberto, cit. (n. 1), p. 10.

${ }^{9}$ Así consta en OIT, Informe acerca de las medidas tomadas para dar cumplimiento a las resoluciones adoptadas por la Conferencia de Santiago de Chile. Segunda Conferencia de los Estados Miembros de la Organización Internacional del Trabajo, La Habana (Ginebra, 1939), p. 3.

${ }^{10}$ Como se aprecia en la convocatoria registrada en las actas. Véase OIT, Resoluciones aprobadas en la Conferencia. Conferencia del trabajo de los estados de América miembros de la Organización Internacional del Trabajo, Santiago (Chile), 2-14 Enero de 1936, Actas, Apéndice VII (OIT, Ginebra), p. XV.

${ }^{11}$ OIT, La Organización Internacional del Trabajo. Lo que es y lo que hace (Oficina 
La Conferencia regional realizada Chile, que fue la primera en su género, revistió una gran importancia y constituyó una novedad en la extensión geográfica y especializada de la Organización Internacional del Trabajo ${ }^{12}$. Reunida, como se verá, a comienzos de 1936, su denominación oficial fue: Conferencia del Trabajo de los Estados de América Miembros de la Organización internacional del Trabajo $^{13}$, y así figura a la cabeza del libro que la reseña ${ }^{14}$.

La convocatoria a la conferencia realizada por el director de la OIT de la época, señor Buttler, se refirió a esta primera conferencia en los siguientes términos: "Tengo el honor de informarle que en el curso de su 72.a sesión, celebrada en Ginebra el 21 de junio de 1935, el Consejo de Administración de la Oficina internacional del Trabajo, me ha encomendado convocar una Conferencia del Trabajo de los Estados de América, Miembros de la Organización, que se abrirá el 30 de diciembre de 1935.

A raíz de una invitación formulada por el primer delegado del gobierno de Chile a la XIX sesión de la Conferencia Internacional del Trabajo, que acaba de celebrarse en Ginebra y que fue recibida con beneplácito por los representantes de los otros Estados de América en esa Conferencia, la Conferencia en cuestión se efectuará en Santiago de Chile. El gobierno de Chile se propone por lo demás, reiterar esta invitación a cada uno de los Estados interesados y, en particular, a aquellos que no se hallaban representados en la XIX Sesión de la Conferencia Internacional del Trabajo" ${ }^{15}$.

En una primera instancia, como se constata, la reunión había sido planeada para comenzar el día 30 de diciembre de 1935. Sin embargo, en una misiva enviada por el director general solicita postergar el inicio de las sesiones para el día 2 de enero de 1936. Es por ello que muchos documentos sitúan a la conferencia en ambos años ${ }^{16}$.

De esta manera, la conferencia se realiza entre los días 2 y 14 de enero de 1936. Los idiomas de trabajos fueron inglés, francés, portugués y, por supuesto, español. ${ }^{17}$ En cuanto a la composición de la conferencia, ésta sería la de delegaciones tripartitas, con dos representantes por cada gobierno, un representante patronal y uno por los obreros. Pudiendo, en todo caso, cada estamento ser asistido por consejeros técnicos, pero tan solo los delegados tendrían derecho a voto ${ }^{18}$. Se siguió

Internacional del Trabajo, Ginebra, 1938), p. 40.

${ }^{12}$ Ibíd., p. 41.

${ }^{13}$ Ibíd.

${ }^{14}$ Conferencia del Trabajo de los Estados de América Miembros de la Organización Internacional del Trabajo. Santiago de Chile, 2 al 14 de enero de 1936 (Oficina Internacional del Trabajo Ginebra, 1936), un volumen de XXXII + 410 pp.

${ }^{15}$ OIT, Resoluciones aprobadas, cit. (n. 10), p. XV.

${ }^{16}$ Véase los documentos de la OIT incluidos en la bibliografía.

${ }^{17}$ OIT, Proposed standing orders, Conference of American States Members of the International Labour Organisation (1st: 1935-1936: Santiago, Chile) (International Labour Office. Geneva: ILO, 1935).

${ }^{18}$ OIT, Resoluciones aprobadas, cit. (n. 10), p. XV. 
entonces la misma estructura que posee desde su creación la Conferencia Internacional del Trabajo ${ }^{19}$. Se hizo una réplica de la estructura mundial a nivel regional.

En este sentido la misma OIT en una de sus publicaciones posteriores a la conferencia de 1936 ha señalado: "La Conferencia de Santiago, en efecto, no tuvo la categoría constitucional de la Conferencia internacional del Trabajo que se reúne por lo menos una vez al año, casi sin excepción, en Ginebra, ni siquiera la de su sección marítima. No equivale a la de Washington de 1919 ni a la de Génova de 1920. No obstante, si no por su extensión, pues se limitó a reunir a los Estados de América Miembros de la Organización Internacional del Trabajo, por la proporcionalidad de los componentes de su representación tripartita -dos delegados gubernamentales, un delegado obrero y un delegado patronal por Estado-y por los trámites internos de su proceso se asemejó mucho a las reuniones generales de la Conferencia"20.

Con el objeto de asegurar el carácter universal ${ }^{21}$ de la OIT, el Consejo de Administración decidió hacerse representar en la Conferencia de Santiago por una delegación compuesta por el presidente del Consejo de Administración y dos miembros de cada uno de los tres grupos, gubernamental, patronal y obrero ${ }^{22}$.

En cuanto al financiamiento de la Conferencia se acordó que cada Estado financiara sus delegaciones y consejeros técnicos. Gran parte de los gastos fueron asumidos por el Estado chileno y eventualmente los gastos extraordinarios podrían ser asumidos por la $\mathrm{OIT}^{23}$. De esta manera, se llegó a un acuerdo en relación al financiamiento, lo que permitió que el Consejo de administración de la oficina hiciera la convocatoria a la Conferencia, decidiera el orden del día y aprobara un presupuesto. Esta manera de proceder hace que la Conferencia de 1936 en Chile se asemeje $\mathrm{e}^{24}$ bastante a la forma cómo se acuerda llevar a cabo anualmente la Conferencia Internacional del Trabajo ${ }^{25}$.

Un aspecto que la diferencia de la Conferencia Internacional del Trabajo, es que en la reunión en Santiago no se podría adoptar ningún instrumento internacional del trabajo, es decir, convenio y recomendaciones ${ }^{26}$. Ello debido a que la constitución de la OIT faculta tan solo a la Conferencia Internacional del Trabajo como órgano legislador internacional.

\footnotetext{
${ }^{19}$ Revisar, Arellano Ortiz, Pablo, La conformidad, cit. (n. 2): Walker Errázuriz, Francisco y Arellano Ortiz, Pablo, Derecho de las relaciones laborales, cit. (n. 2); OIT, Las reglas del juego, cit. (n. 2).

${ }^{20}$ OIT, La Organización Internacional, cit. (n. 11), p. 40.

${ }^{21}$ Según se manifiesta en OIT, Resoluciones aprobadas, cit. (n. 10), p. XV.

${ }^{22} \mathrm{El}$ Consejo de Administración designó a los siguientes representantes por grupo. Por el grupo gubernamental: señor Leggett, representante de Gran Bretaña y al señor Ruiz Manent, representante de España. Por el grupo patronal: los señores Oersted (Dinamarca) y Curein (Yugoeslavia), suplente al Sr. Tzaut (Suiza). Por el grupo obrero: los señores Jouhaux (Francia) y Hayday (Gran Bretaña), y como suplentes los señores Mertens (Bélgica) y Johanson (Suecia).

${ }^{23}$ OIT, Resoluciones aprobadas, cit. (n. 10), p. XVI.

${ }^{24}$ Expresión utilizada por la propia OIT, en OIT, La Organización Internacional, cit. (n. 11), p. 41.

${ }^{25}$ Ver al respecto, Walker Errázuriz, Francisco y Arellano Ortiz, Pablo, Derecho de las relaciones laborales, cit. (n. 2).

${ }^{26}$ OIT, La Organización Internacional, cit. (n. 11), p. 41.
} 
Se registraron delegaciones de "diecinueve Estados, de los veintiuno de América que pertenecen a la Organización: Argentina, Bolivia, Brasil, Canadá, Chile, Colombia, Cuba, República Dominicana, Ecuador, Estados Unidos de América, Guatemala, Haití, México, Nicaragua, Panamá, Paraguay, Perú, Uruguay y Venezuela, Faltaron Honduras y El Salvador. En cambio, Costa Rica, el único Estado de América no Miembro, había enviado un observador gubernamental"27. Aunque de estas delegaciones tan sólo diez fueron completas ${ }^{28}$, es decir, con los tres estamentos del tripartismo: gobiernos, patronos y obreros.

Desde la perspectiva de Chile la Conferencia de 1936 resulto ser también un punto importante dentro de la política internacional que se encontraba en desarrollo. La influencia de la OIT en la naciente legislación social chilena ha sido recogida por la doctrina ${ }^{29}$. De la misma manera se ha señalado que Chile es el primer país latinoamericano en ratificar las primeras normas internacionales del trabajo adoptadas en los inicios de la OIT ${ }^{30}$. Cabe "recordar que las leyes sociales de 1924 contenían muchos de los principios aprobados" 31 en los primeros convenios de la OIT. A este se debe sumar que el director de la Oficina Internacional del Trabajo, Albert Thomas, visita nuestro país en agosto de $1925^{32}$. Es por ello que no resulta extraño que la Conferencia en estudio tenga lugar en nuestro país.

Cabe agregar, además, dentro de la historia de la celebración de esta conferencia la injerencia de un destacado abogado Moisés Poblete Troncoso ${ }^{33}$, quien, como ya señaláramos, se desempeñó como director de la Oficina del Trabajo entre 1920 y 1925. Coincidiendo dicho periodo con la visita de Albert Thomas, con quien, se ha sostenido, que desarrollo una cierta cercanía, calificada incluso de amistad ${ }^{34}$. No es sino que hasta el año 1927 que Poblete llega a Ginebra a la OIT, pero tan solo en 1929 logra un puesto más estable en la estructura de la organización ${ }^{35}$. Entre sus principales logros en Ginebra está una gran labor de compilador de legislaciones sociales de Latinoamérica ${ }^{36}$. Pero, sus funciones no se limitaron a solo recopilar. Tal como se había desempeñado en Chile, logró crear

\footnotetext{
${ }^{27}$ Ibíd.

${ }^{28}$ Ibíd.

${ }^{29}$ Véase YÁñez Andrade, Juan Carlos, La OIT en América del Sur. El comunismo y los trabajadores chilenos (1922-1932) (Santiago, Editorial UAH, 2016); YÁN̄EZ ANDrade, Juan Carlos, Chile y la Organización, cit. (n. 5).

${ }^{30}$ Ibíd., p. 331.

${ }^{31}$ Ibíd., p. 330.

${ }^{32}$ Ibíd., p. 330.

${ }^{33}$ Sobre la obra de este abogado véase Yáñez Andrade, Juan Carlos, Moisés Poblete Troncoso y su aporte a la recopilación de la legislación laboral, Revista de Estudios Histórico-Jurídicos, 40 (2018), pp. 371-390; Él Mismo, Moisés Poblete Troncoso y la primera Historia del movimiento obrero latinoamericano (1946), en Izquierdas, 37 (diciembre 2017), pp. 261-280.

${ }^{34}$ Yáñez Andrade, Juan Carlos, Moisés Poblete Troncoso y su aporte, cit. (n. 33), p. 375.

${ }^{35}$ Sobre los acontecimientos en relación a la contratación de Poblete en la OIT véase YÁÑEZ Andrade, Juan Carlos, Moisés Poblete Troncoso y su aporte, cit. (n. 33), pp. 376-377.

${ }^{36}$ Por ejemplo: Poblete Troncoso, Moisés, La legislación social de América Latina (Ginebra, OIT, 1928-1929), I-II; ÉL MISMO, La aplicación de la legislación social en la América Latina (Ginebra, OIT, 1935); ÉL MISMO, Condiciones de vida y de trabajo de la población indígena del Perú (Ginebra, OIT, 1938).
} 
una buena red de contactos en América Latina realizando diversas misiones que lo posicionaron como la mejor opción para ser el funcionario que se encargaría de apoyar a la Conferencia de 1936 desde Ginebra. Esto se puede reflejar en palabras de Yañez Andrade cuando afirma que "Una vez instalado en Ginebra, y pese a las restricciones presupuestarias que impuso la crisis económica de 1929, Poblete viajó a Chile en 1930, donde realizó una serie de conferencias y tomó contactos con distintas autoridades, además de redactar informes sobre el gobierno del general Carlos Ibáñez del Campo. Recién en 1934 tuvo la posibilidad de participar en una nueva misión a latinoamericana, en el marco de los estudios y asesoría sobre las instituciones de previsión social y la reorganización del Ministerio del Trabajo de Cuba, donde recibió elogiosos comentarios por su labor. Sin embargo, fue la convocatoria a la Primera Conferencia Interamericana del Trabajo de 1936 la que permitió demostrar sus cualidades organizativas. Esta Conferencia se realizó por la invitación del gobierno de Chile durante el transcurso de la Conferencia Internacional del Trabajo de Ginebra en 1935, mostrándose interesado el gobierno de Arturo Alessandri (1932-1938) en consolidar sus relaciones con la OIT luego de la muerte de Albert Thomas en 1932. Por otra parte, el rol asignado a Poblete en la organización de esta Conferencia permite entender que con este evento se cerraba un primer ciclo de vinculaciones internacionales iniciado en 1920, con los primeros contactos entre el mismo Poblete, Arturo Alessandri y Albert Thomas, no siendo extraño que estas antiguas vinculaciones expliquen la disposición de la OIT a convocar una primera Conferencia Interamericana del Trabajo en la ciudad de Santiago de Chile" 37 .

Como se puede apreciar hay un doble movimiento que lleva a que Chile pueda, de manera exitosa, proponer y realizar esta primera conferencia regional en la historia de la OIT. Por un lado, el compromiso del gobierno chileno el cual se demuestra en acciones concretas, una nueva legislación social en 1925 y además en la ratificación de los primero convenios adoptados a inicios de la OIT. Por otro lado, la influencias desde adentro de la OIT realizada por Moisés Poblete, en su calidad de especialista en derecho del trabajo latinoamericano.

\section{LA DISCUSIÓN Y SUS RESULTADOS POSTERIORES}

Una segunda cuestión importante en relación al objeto de este trabajo es la temática tratada por la Conferencia. Como señalamos, la orden del día de los temas a discutir fue aprobada por el Consejo de Administración. En ella figuraron dos puntos: 1. Examen, desde el punto de vista de la ratificación y de la aplicación, de la situación de los convenios internacionales del trabajo existentes, ateniéndose particularmente a los convenios y recomendaciones referentes a las cuestiones de seguros sociales y a los convenios y recomendaciones sobre las condiciones de trabajo de las mujeres y de los niños. 2. Examen de las cuestiones susceptibles de ser ulteriormente objeto de discusiones en la Conferencia Internacional del Trabajo.

De una manera general se ha indicado que "dentro del apartado 1, la Confe-

${ }^{37}$ Yáñez Andrade, Juan Carlos, Moisés Poblete Troncoso y su aporte, cit. (n. 33), p. 377. 
rencia adoptó conclusiones muy detalladas sobre el trabajo de las mujeres y de los niños y sobre los seguros sociales. Asimismo una resolución relativa a la ratificación y aplicación de los convenios y recomendaciones en los países americanos. Dentro del apartado 2, una serie de resoluciones muy varias e importantes marcaron la orientación que América quería dar -y, cuando no, que anhelaba América que le dieran- a los problemas del trabajo en los claros y anchurosos ámbitos de sus dominios" 38 .

En relación al punto 1 de la Orden del día la Oficina internacional del trabajo se prepararon una seria de informes ${ }^{39}$. Obrando la Oficina Internacional del Trabajo de la misma manera que como lo hace hasta nuestros días, bridando apoyo técnico a las discusiones de la Conferencia Internacional del Trabajo.

Como se ha señalado el trabajo realizado por los actores sociales, empleadores, trabajadores y Gobiernos, durante esta conferencia fue muy fructífero. De hecho se discutieron y aprobaron 23 resoluciones ${ }^{40}$. Las temáticas de las resoluciones

${ }^{38}$ OIT, La Organización Internacional, cit. (n. 11), pp. 42-43.

${ }^{39}$ Estos informes fueron por ejemplo los siguientes: OIT, Report on social insurance, Conference of American States Members of the International Labour Organisation (1st: 19351936: Santiago, Chile) (International Labour Office, Geneva: ILO, 1935); OIT, Report on the applications of Conventions ratified by the American States, Conference of American States Members of the International Labour Organisation (1st: 1935-1936: Santiago, Chile) (International Labour Office. Geneva: ILO, 1935); OIT, Report on the conditions of work of women, Conference of American States Members of the International Labour Organisation (1st: 1935-1936: Santiago, Chile) (International Labour Office. Geneva: ILO, 1935); OIT, Report on the employment of children and young persons, Conference of American States Members of the International Labour Organisation (1st 1935-1936: Santiago, Chile) (International Labour Office. Geneva: ILO, 1935).

${ }^{40}$ Las resoluciones aprobadas fueron: 1) resolución relativa a la ratificación y aplicación de las convenciones y recomendaciones en los países americanos (propuesta por el señor Ramírez Otárola, delegado patronal del Perú); 2) resolución relativa a la inmigración (propuesta por el señor Unsain, delegado gubernamental de la Argentina); 3) resolución relativa a la formación, en cada país, de organizaciones centrales, patronales y obreras (propuesta por el grupo obrero); 4) resolución relativa a varias cuestiones de orden social (propuesta por el grupo obrero); 5) resolución relativa a las condiciones de vida y de trabajo de las poblaciones indígenas en los países americanos (propuesta por el señor Rebagliati, delegado gubernamental del Perú); 6) resolución relativa a las relaciones de la Organización Internacional del Trabajo en los países de América; 7) resolución relativa a la desocupación; 8) resolución relativa a la reforma del calendario (propuesta por el señor Goyeneche, delegado patronal de Chile); 9) resolución relativa al estudio, en las universidades e institutos docentes, de las relaciones entre el, capital y el trabajo (propuesta por los delegados gubernamentales, patronal y obrero del Ecuador); 10) resoluciones relativas al descanso semanal (propuesta por los delegados gubernamentales, patronal y obrero del Ecuador); 11) resolución relativa a la defensa jurídica de los derechos del asalariado (propuesta por el señor Rebagliati, delegado gubernamental del Perú); 12) resolución relativa a la publicación, en edición económica, de una historia de los orígenes y antecedentes de la Organización Internacional del Trabajo (propuesta por el señor Silva. delegado gubernamental de Haití); 13) resolución relativa al salario mínimo (propuesta por el señor Escríbar, delegado gubernamental de Chile); 14) eesolución relativa al estudio de las condiciones del trabajo en la agricultura (propuesta por la delegación gubernamental de México); 15) resolución relativa al estudio estadístico del costo de la vida en los países: americanos (propuesta por la delegación gubernamental de México); 16) resolución relativa a la alimentación popular (propuesta por la delegación gubernamental de Chile); 17) resolución relativa a la estadística del trabajo agrícola 
pueden clasificarse en a) generales y económicos; b) administración social; c) estadísticas y encuestas; e) jornada; f) salario y mejoras del salario real; y g) política social para los indios ${ }^{41}$. De ellas 5 fueron propuestas por Chile: 3 por el gobierno chileno, 1 por el grupo patronal, 1 por el delegado obrero chileno en conjunto con el gobierno del Perú.

Las resoluciones adoptadas son de una diversidad y profundidad importante. Ellas tienen un impacto en el trabajo futuro de la organización y sus funcionarios. Así por ejemplo en relación a las funciones de Moisés Poblete, Yañez Andrade nos señala que "Las resoluciones de la Conferencia Interamericana del Trabajo de 1936 tuvieron dos consecuencias concretas en las tareas que Poblete realizaba en la institución de Ginebra. En primer lugar, se recomendó al Consejo de Administración de la OIT la realización de diversos estudios sobre las condiciones de trabajo en la agricultura y la situación de la población indígena. En este último caso, se le asignó a Poblete la tarea de llevar a cabo una investigación sobre la condición indígena en el Perú, la cual fue publicada en 1938. Uno de los aportes principales de este estudio fue determinar la cantidad de indígenas que habitaban el país y sus modalidades de producción en la agricultura e industria. Además, propuso una serie de medidas legales y educativas, con el fin de evitar las formas de trabajo compulsivo y lo extendido que estaba el uso del alcohol y de la coca entre la población indígena. En segundo lugar, la Conferencia Interamericana del Trabajo fue la instancia para que los delegados expresaran una serie de demandas en orden a acercar aún más las tareas de la OIT al continente, obligando a crear nuevas oficinas de corresponsales, entre ellas una en Santiago de Chile”ł2.

El mismo autor indica que obviamente Moisés Poblete pareció ser el funcionario más calificado de la OIT en materias regionales americanas. Por tanto, su traslado a América del Sur, que fue decidido a comienzos de 1937, "suponía 'proseguir las investigaciones sobre las condiciones de vida y de trabajo de los indígenas en ciertos países y para cumplir otras tareas', como lo confidenció el mismo Poblete en una carta. En ella proponía una estadía de dos años en el cargo de corresponsal en Santiago, pero realizando las mismas funciones en países vecinos. Esta estadía, que se transformó en definitiva le permitió desde Chile continuar con sus responsabilidades de elaborar informes sobre la región y participar en distintas reuniones internacionales, como la Conferencia Interamericana del Trabajo de la

en los países de América (propuesta por la delegación gubernamental de México); 18) resolución relativa a la creación de los órganos administrativos y técnicos necesarios para la aplicación real de la legislación del trabajo (propuesta por la delegación gubernamental de Chile); 19) resolución relativa a la alimentación popular (texto refundido de los proyectos de resolución presentados por el señor Llosa, delegado gubernamental del Perú y por el señor Solís, delegado obrero de Chile); 20) resolución relativa a la coordinación de la política económica de los Estados y la legislación protectora de los trabajadores (propuesta por la delegación gubernamental de Haití); 21) resolución relativa a los monopolios particulares (propuesta por la delegación de Haití); 22) resolución relativa al Truck System (propuesta por el señor Forn, delegado gubernamental de la Argentina); 23) resolución relativa a los seguros sociales y al trabajo de las mujeres y de los niños. Véase al respecto, OIT, Resoluciones aprobadas, cit. (n. 10).

${ }^{41}$ OIT, La Organización Internacional, cit. (n. 11), pp. 43-44.

${ }^{42}$ Yáñez Andrade, Juan Carlos, Moisés Poblete Troncoso y su aporte, cit. (n. 33), p. 378. 
Habana de 1939 y la Conferencia Internacional del Trabajo de Nueva York de 1941 "43. Sin lugar a dudas, La realización de esta Conferencia y las actividades de Poblete en el seno de la OIT lograron posicionar a Chile dentro de la discusión internacional de las normas de la OIT.

En cuanto al contenido de las resoluciones ellas han tenido un variado grado de impacto en las actividades de la OIT. Nos gustaría referirnos brevemente en particular a tres temas que consideramos aun relevantes, en relación a los efectos que este evento histórico ha tenido en la organización y en las normas internacionales del trabajo adoptadas por la OIT, que fueron discutidos en las décima, undécima y duodécima sesiones de la Conferencia.

\section{Los seguros sociales}

El primero de los temas que hemos escogido dice relación con la discusión en relación a los seguros sociales. La cual se fijó de la siguiente manera: "Resoluciones relativas a los seguros sociales y al trabajo de las mujeres y de los niños. Los textos de las resoluciones elaboradas por las dos comisiones técnicas de la Conferencia y que fueron aprobados por ésta en sus undécima y decimosegunda sesiones plenarias, no se reproducen aquí por figurar en los respectivos informes de comisión". El texto al cual se hace referencia es bastante detallado y técnico ${ }^{44}$.

Se ha considerado acertadamente a nuestro parecer que esta "resolución marca una nueva etapa en la evolución de los seguros sociales en América. Ha servido para exponer claramente las necesidades y aspiraciones del nuevo continente, habiendo contribuido su adopción a acelerar y también a dar más firmeza al desarrollo de los regímenes de los seguros sociales" ${ }^{35}$. E incluso podríamos decir que marca una etapa en la evolución de la seguridad social a nivel mundial por el impacto que esta discusión tiene en la adopción de dos recomendaciones: la recomendación sobre la seguridad de los medios de vida, 1944 (núm. 67) ${ }^{46}$ y recomendación sobre la seguridad social (fuerzas armadas), 1944 (núm. 68) ${ }^{47}$. De hecho, en el preámbulo de la recomendación $67^{48}$ se destaca las resoluciones

${ }^{43}$ Ibíd.

${ }^{44} \mathrm{El}$ texto se puede consultar en OIT, Resoluciones aprobadas, cit. (n. 10), p. 333.

${ }^{45} \mathrm{OIT}$, Informe acerca de las medidas tomadas para dar cumplimiento a las resoluciones adoptadas por la Conferencia de Santiago de Chile. Segunda Conferencia de los Estados Miembros de la Organización Internacional del Trabajo, La Habana, (Ginebra, 1939), p. 18.

${ }^{46}$ Consultar texto en: https://www.ilo.org/dyn/normlex/es/f?p=NORMLEXPUB:12100: 0::NO:12100:P12100_INSTRUMENT_ID:312405:NO

${ }^{47}$ Consultar texto en: https://www.ilo.org/dyn/normlex/es/f?p=NORMLEXPUB:12100: 0::NO:12100:P12100_INSTRUMENT_ID:312406:NO

${ }^{48}$ Extracto "Considerando que la Organización Internacional del Trabajo ha promovido el desarrollo de la seguridad de los medios de vida: mediante la aprobación por la Conferencia Internacional del Trabajo de convenios y recomendaciones sobre la indemnización por accidentes del trabajo y enfermedades profesionales, el seguro de enfermedad, las prestaciones de maternidad, las pensiones de vejez y de invalidez, las pensiones de viudedad y de orfandad y las prestaciones a los trabajadores desempleados; mediante la aprobación por la primera y la segunda Conferencia del Trabajo de los Estados de América Miembros de la Organización Internacional del Trabajo de las resoluciones que constituyen el Código Interamericano de Seguros Sociales; la participación de una delegación del Consejo de Administración en la primera Conferencia Interamericana de 
aprobadas en la primera y la segunda Conferencia del Trabajo de los Estados de América Miembros de la Organización Internacional del Trabajo, es decir en la reunión de Santiago en 1936 y en La Habana en 1939, como parte del contenido que motiva su adopción.

La discusión en la Conferencia de Santiago de 1936 se llevó a cabo en la Comisión de Seguros Sociales la cual tuvo seis sesiones en la que se discutieron aspectos de los seguros de accidentes, enfermedades, invalidez, vejez y muerte ${ }^{49}$, utilizando como base para dicha discusión el informe presentado por la Oficina Internacional del Trabajo ${ }^{50}$. Luego de largas explicaciones de los miembros de la comisión de seguros sociales sobre cada uno de los riesgos sociales estudiados por la comisión, el presidente de la comisión solicita a los miembros de la Conferencia adoptar la resolución en vista que en la comisión este fue adoptado por unanimidad. De esta manera el presidente de la Conferencia propone su votación y se adopta la resolución ${ }^{51}$.

El informe presentado por la Oficina Internacional del Trabajo a la Conferencia de la Habana contiene sendos pasajes para referirse a los avances en relación a esta resolución. Dicho informe se encuentra dividido en tres partes. De ellas la primera parte está casi completamente destinado a informar el avance de la legislación social en esta materia ${ }^{52}$.

Quisiéramos mencionar que el estudio realizado en esta Conferencia ha servido de base para la adopción de una norma internacional del trabajo de carácter vinculante, Nos referimos al Convenio sobre la seguridad social (norma mínima), 1952 (núm. 102) (3) $^{3}$ el cual se considera como el texto base para el establecimiento de un sistema de seguridad social y que fue adoptado siguiendo los lineamientos de las recomendaciones núm. 67 y $68^{54}$. Cabe mencionar entonces la importante influencia de esta reunión en el nacimiento y evolución de la seguridad social como rama del derecho. En este sentido la región latinoamericana ha sido señalada como una de las regiones en donde los seguros sociales tienen un origen temprano. Más aun se ha señalado a la legislación chilena y brasileña como pioneras en la región ${ }^{55}$.

El informe presentado sobre esta materia y su discusión en la Conferencia en

Seguridad Social, que adoptó la Declaración de Santiago de Chile, y la aprobación por el Consejo de Administración de los Estatutos de la Conferencia Interamericana de Seguridad Social, que ha sido establecida como organismo permanente de cooperación entre las administraciones e instituciones de seguridad social y actúa de concierto con la Oficina Internacional del Trabajo; y mediante la participación de la Oficina Internacional del Trabajo, como organismo consultor, en la elaboración de sistemas de seguridad social, en diversos países, y a través de otras medidas". Lo destacado es nuestro.

${ }^{49}$ Como consta en la intervención del presidente de la Comisión de Seguros Sociales, señor Sandoval, ver OIT, Resoluciones aprobadas, cit. (n. 10), p. 236.

${ }^{50}$ A saber OIT, Report on social insurance, cit. (n. 39).

${ }^{51}$ Como consta en OIT, Resoluciones aprobadas, cit. (n. 10), p. 257.

${ }^{52}$ Véase OIT, Informe acerca de las medidas tomadas, cit. (n. 44).

${ }^{53}$ Cuyo texto se puede consultar en https://www.ilo.org/dyn/normlex/es/f?p=NORMLEX PUB:12100:0::NO:12100:P12100_INSTRUMENT_ID:312247:NO

${ }^{54} \mathrm{Al}$ respecto ver Arellano OrTíz, Pablo, Lecciones de seguridad social (2a ed., Librotecnia, Santiago, junio 2017).

${ }^{55}$ Durand, Paul, La politique contemporaine de sécurité sociale (Paris, Dalloz, 2005), p. 85. 
Santiago, así como también su posterior influencia en la adopción de las recomendaciones y convenio citados, demuestran que la Conferencia de Santiago de 1936 tiene un claro rol determinante en el proceso de evolutivo de la seguridad social.

\section{Los derechos de los pueblos indigenas}

En relación con los derechos de los pueblos indígenas la resolución adoptada indica: "Resolución relativa a las condiciones de vida y de trabajo de las poblaciones indígena en los países americanos. Propuesta por el señor Rebagliati, delegado gubernamental del Perú. La Conferencia del Trabajo de los Estados americanos Miembros de la Organización Internacional del Trabajo, recomienda al Consejo de Administración de la Oficina internacional del Trabajo: 1) Que se dirija a todos los países del Continente americano cuya población, de modo importante, esté constituida por núcleos de población indígena, solicitando que comuniquen a dicha Oficina cuantas observaciones se juzguen pertinentes y cuanta documentación esté a su alcance y que guarden relación con los problemas económicos y sociales de vida y de trabajo de dicha población. 2) Que imparta instrucciones a la Oficina Internacional del Trabajo con objeto de que se inicie un estudio especial de este problema y que se tomen en cuenta las posibilidades que existan para llegar a una acción internacional determinada con un objeto práctico" 56 .

La propuesta presentada por el delegado del gobierno del Perú resulta curiosa para el momento de la reunión. Sin embargo, como consta en las actas de la Conferencia, en la décima sesión cuando el presidente ofrece la palabra ningún delegado formuló observaciones dándose por aprobada la propuesta ${ }^{57}$. Cabe entonces preguntarse por qué se considera a nuestro parecer relevante esta resolución. Pues bien, ella pretende obtener información por parte de los Estados americanos que permitan poder estudiar en mayor profundidad la situación de la población indígena. Ello se hace con cautela incluyendo que lo se busca son datos sobre sus problemas económicos, sociales y relativos al trabajo. De esta manera se puede apreciar un temprano interés en proteger, dentro del contexto de la OIT, a los pueblos indígenas.

A nuestro entender es en esta conferencia en donde se inicia el interés de la organización por los temas indígenas. Tenemos claro que no es hasta varios años más tarde que se adoptan el Convenio sobre poblaciones indígenas y tribuales, 1957 (núm. 107) ${ }^{58}$ y la Recomendación sobre poblaciones indígenas y tribuales, 1957 (núm. 104), y posterioridad a estos se adopta Convenio sobre pueblos indígenas y tribales, 1989 (núm. 169) ${ }^{59}$.

Sin embargo, en la Conferencia de La Habana al darse cuenta del estado de avance en relación a las resoluciones de la Conferencia de Santiago se indica por ejemplo que "Un estudio sobre las condiciones de vida y de trabajo de los

\footnotetext{
${ }^{56}$ OIT, Resoluciones aprobadas, cit. (n. 10).

${ }^{57}$ Ibíd., p. 223.

${ }^{58}$ Consultar texto en: https://www.ilo.org/dyn/normlex/es/f?p=NORMLEXPUB:12100: 0::NO::P12100_ILO_CODE:C107

${ }^{59}$ Consultar texto en: https://www.ilo.org/dyn/normlex/es/f?p=NORMLEXPUB:12100:: :NO:12100:P12100_ILO_CODE:C169:NO
} 
trabajadores indígenas en el Perú fue publicado en Santiago de Chile y fue publicado igualmente en español el informe sobre la Conferencia de migraciones colonizadoras que tuvo lugar en Ginebra y a la cual asistieron los representantes de los países que se interesan particularmente en los problemas migratorios"60. Además, se informa que "con posterioridad a la Conferencia de Santiago (desde febrero hasta abril de 1936) un funcionario chileno de la Oficina llevó a cabo en el Perú, en Bolivia y en el Ecuador un viaje de encuesta sobre las condiciones de trabajo de los indígenas, buscando los elementos preparatorios del estudio ya proyectado por la Conferencia de Santiago sobre las condiciones de trabajo y de vida de las poblaciones indígenas" 61 . En el mismo sentido, "en febrero y marzo de 1937, un funcionario mexicano de la Oficina visitó su país para emprender un estudio preliminar sobre las condiciones de trabajo y de vida de la población indígena y para obtener informaciones sobre la evolución de los problemas agrícolas en México" ${ }^{2}$.

En cuanto a la resolución misma, el informe presentado a la Conferencia de la Habana da cuenta que el "Consejo de administración atendió al primero de los deseos formulados por la Conferencia de Santiago. Además ha encargado a la Oficina que emprenda el estudio solicitado" ${ }^{63}$. En el mismo informe se agrega: "Aunque la Oficina Internacional del Trabajo había procedido, algún tiempo atrás, al estudio de diversos aspectos de los problemas tocantes al trabajo indígena, no tuvo hasta entonces la oportunidad de incluir a las poblaciones indígenas de los países de América en el campo de sus estudios. Ahora bien, las condiciones de vida y de trabajo de los indígenas de estos países son netamente diferentes de las de las poblaciones indígenas de los demás continentes. Por otra parte, los problemas etnográficos, económicos, sociales, etc., debidos a la existencia en cierto número de países americanos de núcleos importantes de poblaciones indígenas, presentan diferencias muy marcadas según el país de que se trate. Era necesario, pues, antes de emprender los estudios pedidos por la Conferencia de Santiago, efectuar investigaciones preliminares a fin de darse cuenta de la amplitud del problema y poder escoger el mejor método para llevar a cabo la encuesta" ${ }^{34}$.

Se da cuenta, además, que desde la Conferencia de Santiago, la Oficina se ocupó de agrupar la documentación que pudiera servir de base al estudio proyectado. Si bien se trataba de un tema nuevo y cuya preparación sería difícil, se logró publicar un libro en corto tiempo. De esta manera se hace referencia en el informe al libro titulado "Condiciones de vida y de trabajo de la población indígena del Perú”, preparado por Moisés Poblete Troncoso y publicado por la Oficina Internacional del Trabajo en $1938^{65}$.

${ }^{60} \mathrm{OIT}$, Informe acerca de las medidas tomadas, cit. (n. 44), p. 155.

${ }^{61}$ Ibíd., p. 157.

${ }^{62}$ Ibíd., pp. 157-158.

${ }^{63}$ Ibíd., p. 174.

${ }^{64}$ Ibíd., pp. 174-175.

${ }^{65}$ Ibíd., p. 175. De ello también da cuenta la doctrina, revisar YÁ̄́nez ANDRADE, Juan Carlos, La Organización Internacional del Trabajo y el problema social indígena: La encuesta en Perú de 1936, en Secuencia, 98 (mayo-agosto 2017), pp. 130-157. 
El ejercicio propuesto en la resolución de la Conferencia de Santiago "hizo que la Oficina Internacional del Trabajo llegase a la conclusión de que en adelante debería concentrar sus esfuerzos para lograr que todos los gobiernos de los Estados americanos enviasen una detallada información sobre las condiciones de vida y de trabajo de sus poblaciones indígenas de manera que fuese posible hacer un estudio general comparativo de los problemas planteados. Con este objeto, la Oficina se ha dirigido a los miembros americanos de la Organización Internacional del Trabajo, pidiéndoles que remitan, de acuerdo con un plan general que se ha establecido, la información a que hace referencia la primera parte de la resolución de Santiago. Tan pronto como se tenga una información detallada, la Oficina podrá hacer un progreso más rápido en lo que se refiere a la coordinación de la encuesta, teniendo presente los propósitos e intenciones del segundo apartado de la resolución de Santiago" ${ }^{66}$.

No cabe duda a nuestro parecer que el posicionamiento del tema indígena tiene dentro de la OIT un origen germinal en la Conferencia de Santiago de 1936. Por último, en relación a esta temática cabe señalar que en 2019, el año del centenario de la OIT coincide con el $30^{\circ}$ aniversario de la adopción del Convenio sobre pueblos indígenas y tribales, 1989 (núm. 169) por parte de la Conferencia Internacional del Trabajo. Cabe indicar además que de entre los países que han ratificado este convenio un número importante son latinoamericano ${ }^{67}$. Dentro de los cuales se encuentra nuestro país.

\section{La OIT y los paises de América}

El tercero ámbito que nos gustaría analizar es en relación a la denominada: "Resolución relativa a las relaciones de la Organización Internacional del Trabajo con los países de América"68.

El hecho de realizar una reunión regional tripartita bajo similares características de la Conferencia Internacional del Trabajo pero a nivel regional implicó un gran respaldo a la propuesta del gobierno chileno, así como también a la región latinoamericana. Es por ello que una resolución que hiciera referencia a la región no fue algo extraño.

La propuesta fue hecha por el delegado del gobierno de Uruguay. Su intención fue poder sentar las bases para el trabajo de la Organización en la región, pidiendo estudios sobre los temas sociales de los países de la región.

Pero no solo se emite un voto para que el Consejo de Administración revise en detalle las cuestiones que se aprobaron en la Conferencia de Santiago. Además, se indica que se pondrá especial atención a las cuestiones que fueran propuestas por

${ }^{66}$ OIT, Informe acerca de las medidas tomadas, cit. (n. 44), p. 175.

${ }^{67}$ De acuerdo a la información disponible en la página ilo.org (consultada el 19 de junio de 2019 a las 21:00hrs) el número total de ratificaciones del Convenio Núm. 169 son 23. Los países latinoamericanos que han ratificado son 15: Argentina, Estado Plurinacional de Bolivia, Brasil, Chile, Colombia, Costa Rica, Dominica, Ecuador, Guatemala, Honduras, México, Nicaragua, Paraguay, Perú y República Bolivariana de Venezuela. El resto de los países son 8: República Centroafricana, Dinamarca, España, Fiji, Luxemburgo, Nepal, Noruega y Países.

${ }^{68}$ OIT, Resoluciones aprobadas, cit. (n. 10). 
el señor Sandoval, delegado del gobierno de Cuba; por el señor Unsain, delegado gubernamental de Argentina, y por el grupo obrero. Estas cuestiones fueron las siguientes: “a) posibilidad de convocar, en el porvenir, cuando las circunstancias lo hagan parecer oportuno, conferencias periódicas análogas a la Conferencia de Santiago; b) aumento del número de nacionales de los Estados de América en el seno de las comisiones técnicas de la Organización; c) aumento del número de los funcionarios americanos en la Oficina Internacional del Trabajo, reclutándoselos entre las personas más capacitadas y conocedoras del medio; d) aumento de las oficinas de correspondencia y corresponsalías de la Oficina Internacional del Trabajo en los diferentes países de América; e) intensificación de las investigaciones y encuestas documentales emprendidas por la Oficina Internacional del Trabajo en colaboración con los Estados de América sobre los problemas que presentan un interés especial para ellos; f) inclusión de estudios periódicos sobre los hechos y el derecho de América en las publicaciones de la Oficina Internacional del Trabajo, y muy particularmente en su repertorio de jurisprudencia; g) desarrollo y mayor difusión, en los idiomas en uso en el continente americano, de las publicaciones de la Oficina Internacional del Trabajo que presenten un interés particular para los Estados de América, y especialmente publicación de ediciones de divulgación popular a bajo precio; h) preparación por la Oficina Internacional del Trabajo, de acuerdo con un plan sistemático, de una obra que permita conocer y divulgar el esfuerzo cumplido por cada uno de los países de América en el mejoramiento de la vida social continental"69.

En la discusión de esta propuesta de resolución el delegado gubernamental de Uruguay, señor Antuña, recalcó la necesidad de la solidaridad americana, y que ella no era contradictoria con el fin universalista de la OIT ${ }^{70}$. Por su parte, el Sr. Arroyo, de la delegación gubernamental de Ecuador señalo: "Nosotros creemos que la Unión Panamericana está absolutamente incapacitada para afrontar estos problemas técnicos del trabajo; y justa preocupación sería, sobre todo para los elementos obreros. Que fuéramos a confiar estas cuestiones técnicas, que hay que afrontar y resolver en una triple ecuación de gobierno, patrones y obreros, por medio da asociaciones de carácter eminentemente diplomático, en las cuales, por su misma naturaleza, no serían, precisamente, ni el grupo patronal ni menos el grupo obrero los que hubieran de sentirse debidamente representados. No quiero abundar en mayores razonamientos, pero sí ratificar una vez más, que el Ecuador está convencido de que los problemas americanos deben estudiarse para América, no importando que ellos se resuelvan desde otros continentes; pero no deben estudiarse por América para llevarlos a otros tribunales menos capacitados, mientras más aislados de nuestra realidad se encuentren"71.

Por su parte la OIT ha considerado esta resolución como un contenido especial "por la cual se define la colaboración entre la Organización del Trabajo y sus Miembros americanos: convocatoria futura de otras Conferencias como la de Santiago; creación de nuevas corresponsalías de la Oficina de Ginebra en los

\footnotetext{
${ }^{69}$ Ibíd.

${ }^{70}$ Ibíd., pp. 224-227.

${ }^{71}$ Ibíd., p. 229.
} 
países americanos; nombramiento de mayor número de nacionales de éstos en las Comisiones de peritos o en el personal de aquélla; desenvolvimiento de las investigaciones y de los estudios sobre los problemas y las iniciativas en materia social de aquellos países y de las publicaciones en sus idiomas más esparcidos. La Conferencia Panamericana de la Paz, idea del presidente Roosevelt, que tuvo lugar solemnemente en Buenos Aires en 1936, apoyó la política adoptada en la Conferencia de Santiago refiriéndose expresamente a los acuerdos intervenidos respecto de las emigraciones de Europa a América y de las encuestas sobre el nivel de vida y otros factores de la situación económica. En uno y en otro caso, los estudios e investigaciones de la Unión Panamericana se realizarán sin perjuicio de los encomendados a la Oficina Internacional del Trabajo y vendrán a añadirse a los de ésta"72.

Luego de la Conferencia de 1936 en Santiago a nadie le extraño que el señor Butler haya informado al Consejo de administración de la Oficina Internacional del Trabajo sobre la obra de la Conferencia de Santiago. En donde se destaca su carácter esencialmente americano. Así como también se destaca el "gran número de problemas se plantean allí de un modo peculiarísimo. Por ejemplo, los de la inmigración, la cuestión agraria, la política indiana. Esta, en algunos países como en México, está muy adelantada. Pero, en general, y aparte de las condiciones de raza, de clima y de tradición de tan dilatado pedazo del mundo, la circunstancia de tener muchas de sus regiones poblaciones muy diseminadas y heterogéneas y la de que varios Estados americanos no hayan alcanzado todavía un estadio muy avanzado de desenvolvimiento industrial, explican muchas características especialísimas de sus inquietudes. La falta de éxito de las organizaciones profesionales en muchos sitios podría encontrar, en cierto modo, un fundamento en esas circunstancias. En cuanto a la ratificación de los convenios internacionales del Trabajo, parece que algunos Estados de América la consideran como un estimulante muy útil para el progreso de su propia legislación social, y por eso muchas veces los ratifican sin estar seguros de poder aplicarlos. Ya se habló de la importancia que esto tiene. De ahí la necesidad de los debidos acomodamientos pregonados en la Conferencia"73.

Los países americanos hicieron explicito su deseo de tener una mayor injerencia en la organización y en la Oficina Internacional del Trabajo. Su llamado fue escuchado y desde entonces se realizan periódicamente reuniones regionales tripartitas siguiendo el modelo iniciado en la reunión de Santiago de $1936^{74}$.

${ }^{72}$ OIT, La Organización Internacional, cit. (n. 11), pp. 44-45.

${ }^{73}$ Ibíd.

${ }^{74}$ La última de estas reuniones se realizó en Ciudad de Panamá en octubre de 2018, siendo la decimonovena reunión de este tipo realizada en el continente americano. Para más información de este reunión ver: https://www.ilo.org/global/meetings-and-events/regional-meetings/ americas/19amrm/lang--es/index.htm 


\section{REFLEXIONES FINALES}

La importancia de la Conferencia de Santiago de Chile ha sido encarecida debido a que ha abierto un capítulo nuevo en la historia de la Organización Internacional del Trabajo ${ }^{75}$.

El mismo Consejo de Administración de la Organización en su informe en relación a la Conferencia de Santiago se "dio por satisfecho con lo manifestado en el Informe y, haciendo dos partes con las resoluciones de la Conferencia, encargó al director que diera curso inmediato a aquellas que tienden sencillamente a que su texto sea puesto en conocimiento de los Estados interesados, y le invitó, respecto de las otras, a recordárselas a las comisiones competentes y a que él mismo las tuviera muy en cuenta cuando haya de hacer proposiciones relativas a sus asuntos"76.

El existo de la reunión de Santiago en 1936 permitió que, como parte de su seguimiento, se realizara una segunda reunión regional. Esta vez en La Habana, Cuba, en 1939. En dicha reunión la Oficina Internacional del Trabajo presentó un informe de seguimiento de los resuelto en Santiago. En dicho informe se destaca que "Los valiosos contactos establecidos gracias a ella se han dejado sentir no sólo en la labor cotidiana de la Oficina Internacional del Trabajo, sino asimismo en las decisiones de la Conferencia Internacional del Trabajo y del Consejo de administración" 77 . Se indica, además, que "las recomendaciones hechas por la Conferencia de Santiago sobre cuestiones tan diversas se han ido incorporando gradualmente, en el transcurso de los tres años últimos, en la vida de la Organización. En lo que se refiere a la legislación internacional del trabajo, a los seguros sociales, a las migraciones, a los problemas económicos, a las condiciones de trabajo de las mujeres y de los niños, y a otras muchas cuestiones, se habría podido observar que en estos tres años la parte que ha correspondido a los países de América en la actuación de la Organización ha llegado a ser mucho más importante que en el pasado, y que, al mismo tiempo, estos Estados han podido utilizar en forma más completa los servicios que la Organización Internacional del Trabajo puede prestarles"78.

Se destacada que "La Conferencia de Santiago, como primera oportunidad de formular los deseos de los Estados de América, ha sido el punto de partida de una colaboración mucho más sistemática y más comprensiva que la desarrollada hasta entonces. Se ha logrado con ella mucho más de lo que la asistencia de delegaciones de aquellos países a muchas reuniones anuales de la Conferencia Internacional del Trabajo hubiera podido hacer para mostrar lo que los Estados de América esperan de la Organización y las características especiales de los problemas sociales, según se plantean en dichos países. Los contactos directos establecidos en Santiago han podido desarrollarse luego grandemente"79. En el mismo sentido "la Oficina no puede disimular su satisfacción por las favorables circunstancias en que le fue dado

\footnotetext{
${ }^{75}$ OIT, La Organización Internacional, cit. (n. 11), p. 46.

${ }^{76}$ Ibíd., pp. 45-46.

${ }_{77}$ OIT, Informe acerca de las medidas tomadas, cit. (n. 44), p. 232.

${ }^{78}$ Ibíd.

${ }^{79}$ Ibíd.
} 
convocar y celebrar la Conferencia de Santiago en un momento tan oportuno. Ya el hecho de que se adoptara la proposición de convocar la Conferencia constituyó una manifestación de este amplio movimiento, que continúa sin descanso, siguiendo, por el contrario, su desarrollo en línea ascendente" 80 .

Claramente lo realizado en Santiago marca un antes y un después para la OIT. $\mathrm{Y}$ es por ello que, como se indica por la propia OIT, "se ha de reconocer que el progreso logrado, en un espacio de tiempo relativamente corto, es considerable y constituye un buen augurio para el futuro" ${ }^{81}$. Y es por ello que en el informe de seguimiento se llama a seguir avanzando en esta senda, señalando que "A la Conferencia de La Habana corresponde ahora dar nuevo ímpetu a la obra emprendida en Santiago, definiendo los problemas y las aspiraciones que en la hora presente ocupan la atención de los Estados americanos en materia social. Su labor ha de resultar seguramente facilitada por el balance en que la Oficina ha procurado resumir los fructíferos resultados de la Conferencia de Santiago"82.

Compartirnos estas reflexiones hechas en su época sobre la importancia de la reunión de Santiago, ya que no solo es un evento importante dentro de la evolución como institución para la OIT, sino que también marca la influencia de los países latinoamericanos en este contexto y la manera cómo posicionaron temas a nivel mundial.

La propuesta del gobierno chileno de promover un encuentro regional se puede considerar como acertada. Se posiciona así al Estado chileno como promotor de un evento clave en la historia del derecho internacional del trabajo. La Conferencia de 1936 constituye un evento histórico tanto por su contenido político como también por los temas discutidos. A nuestro parecer la discusión llevada a cabo en relación a los seguros sociales y a los pueblos indígenas resulta ser clave para posicionar a la región latinoamericana dentro del contexto internacional.

\section{BiBLIOGRAFÍA}

Arellano Ortiz, Pablo, La conformidad de la legislación chilena a las normas internacionales del trabajo de la OIT, en Revista de Derecho de la Universidad Católica de la Santísima Concepción, 24 (junio 2011), pp. 39-60.

- Lecciones de seguridad social (2a ed., Librotecnia, Santiago, junio 2017).

Boivin, Isabelle y Odero, Alberto, La Comisión de Expertos de la OIT y el progreso de las legislaciones nacionales, en Revista Internacional del Trabajo, 125/3 (2006).

Durand, Paul, La politique contemporaine de sécurité sociale (Dalloz, Paris, 2005).

OIT, Las reglas del juego: Una introducción a la actividad normativa de la Organización Internacional del Trabajo (Ginebra, Oficina Internacional del Trabajo, 2019).

- Segunda Conferencia Interamericana del Trabajo. Memoria del Director (Ginebra, OIT, 1939).

- Informe acerca de las medidas tomadas para dar cumplimiento a las resoluciones adoptadas por la Conferencia de Santiago de Chile. Segunda Conferencia de los Estados

\footnotetext{
${ }^{80}$ Ibíd., p. 234.

${ }^{81}$ Ibíd.

${ }^{82}$ Ibíd.
} 
Miembros de la Organización Internacional del Trabajo, La Habana, (Ginebra, 1939).

- La Organización Internacional del Trabajo. Lo que es y lo que hace (Oficina Internacional del Trabajo, Ginebra, 1938).

- Resoluciones aprobadas en la Conferencia. Conferencia del trabajo de los estados de América miembros de la Organización Internacional del Trabajo, Santiago (Chile), 2-14 Enero de 1936, Actas, Apéndice VII (OIT, Ginebra).

- Conferencia del Trabajo de los Estados de América Miembros de la Organización internacional del Trabajo. Santiago de Chile, 2 al 14 de enero de 1936. Un volumen de XXXII + 410 páginas (Oficina Internacional del Trabajo Ginebra, 1936).

- Report on social insurance, Conference of American States Members of the International Labour Organisation (1st: 1935-1936: Santiago, Chile) (International Labour Office, Geneva: ILO, 1935).

- Report on the applications of Conventions ratified by the American States, Conference of American States Members of the International Labour Organisation (1st: 1935-1936: Santiago, Chile) (International Labour Office. Geneva: ILO, 1935).

- Report on the conditions of work of women, Conference of American States Members of the International Labour Organisation (1st: 1935-1936: Santiago, Chile) (International Labour Office. Geneva: ILO, 1935).

- Report on the employment of children and young persons, Conference of American States Members of the International Labour Organisation (1st: 1935-1936: Santiago, Chile) (International Labour Office. Geneva: ILO, 1935).

- Proposed standing orders, Conference of American States Members of the International Labour Organisation (1st: 1935-1936: Santiago, Chile) (International Labour Office. Geneva: ILO, 1935).

- Bureau international du Travail. Procés-verbaux de la 75 me session du Conseil d'adminislration. Genéve, 23-25 avril 1936 (Geneva, 1936).

Poblete Troncoso, Moisés, La legislación social de América Latina (Ginebra, OIT, 1928-1929), I-II.

- La aplicación de la legislación social en la América Latina (Ginebra, OIT, 1935).

- Condiciones de vida y de trabajo de la población indígena del Perú (Ginebra, OIT, 1938).

Van Der Heijden, Paul, The ILO Stumbling towards Its Centenary Anniversary, en International Organizations Law Review, 15 (2018), pp. 203-220.

Villasmil Prieto, Humberto, La incidencia de la Organización Internacional del Trabajo en el momento fundacional del derecho del trabajo Latinoamericano: unas notas introductorias, en Dialogue Documento de trabajo, núm. 33, Departamento de Relaciones Laborales y de Empleo (Oficina Internacional del Trabajo, Ginebra: OIT, 2011).

Walker Errázuriz, Francisco y Arellano Ortiz, Pablo, Derecho de las relaciones laborales, I: Derecho individual del trabajo (Santiago,Librotecnia, 2016).

Warren, Irvin, Labor Conference of American States, Santiago, Chile, 1936, en Monthly Labor Review (pre-1986), Mar (1936), 42/3.

YÁÑez Andrade, Juan Carlos, Moisés Poblete Troncoso y su aporte a la recopilación de la legislación laboral, en Revista de Estudios Histórico-Jurídicos, 40 (2018), pp. 371-390.

- La Organización Internacional del Trabajo y el problema social indígena: la encuesta en Perú de 1936, en Secuencia, 98 (mayo-agosto 2017), pp. 130-157. 
- Moisés Poblete Troncoso y la primera historia del movimiento obrero latinoamericano (1946), en Izquierdas, 37 (diciembre 2017), pp. 261-280.

- La OIT en América del Sur. El comunismo y los trabajadores chilenos (1922-1932) (Santiago, Editorial UAH, 2016).

- Chile y la Organización Internacional del Trabajo (1919-1925). Hacia una legislación social universal, en Revista de Estudios Histórico-Jurídicos, 22 (2000), pp. 317-332. 